\title{
Alimentação e saúde mental durante a pandemia de Covid-19
}

\author{
Food and mental health during the Covid-19 pandemic \\ Alimentación y salud mental durante la pandemia Covid-19
}

Recebido: 12/10/2021 | Revisado: 22/10/2021 | Aceito: 24/10/2021 | Publicado: 25/10/2021

\author{
Patricia Cristina da Silva Menegotte \\ ORCID: https://orcid.org/0000-0001-8281-2237 \\ Universidade Comunitária da Região de Chapecó, Brasil \\ E-mail: patricia.c.silva@unochapeco.edu.br \\ Catiúscia Göttems Frömming \\ ORCID: https://orcid.org/0000-0002-4757-2300 \\ Universidade Comunitária da Região de Chapecó, Brasil \\ E-mail: catiuscia@unochapeco.edu.br \\ Marcia Orth Ripke \\ ORCID: https://orcid.org/0000-0002-5244-0409 \\ Universidade Comunitária da Região de Chapecó, Brasil \\ E-mail: marciaripke@ unochapeco.edu.br \\ Carla Rosane Paz Arruda Teo \\ ORCID: https://orcid.org/0000-0002-1534-6261 \\ Universidade Comunitária da Região de Chapecó, Brasil \\ E-mail: carlateo@unochapeco.edu.br \\ Vanessa da Silva Corralo \\ ORCID: https://orcid.org/0000-0003-4234-4875 \\ Universidade Comunitária da Região de Chapecó, Brasil \\ E-mail: vcorralo@unochapeco.edu.br \\ Junir Antonio Lutinski \\ ORCID: https://orcid.org/0000-0003-0149-5415 \\ Universidade Comunitária da Região de Chapecó, Brasil \\ E-mail: junir@unochapeco.edu.br
}

\begin{abstract}
Resumo
A pandemia causada pelo Covid-19 ocasionou o isolamento e o distanciamento social, perda de empregos, mudanças na renda familiar, alterações nos hábitos alimentares, bem como, dificuldades de acesso aos alimentos e as imprevisibilidades que afetam a saúde física e emocional de indivíduos e coletividade. Neste contexto, o objetivou-se avaliar os aspectos relacionados à alimentação e à saúde mental da população catarinense durante a pandemia de Covid-19. A coleta de dados foi realizada com a aplicação de um questionário de maneira remota no período de dezembro de 2020 a fevereiro de 2021. O questionário foi composto por questões que abordaram o perfil sociodemográfico, Escala Brasileira de Insegurança Alimentar, Teste - Como está sua alimentação? do Ministério da Saúde, Formulário de marcadores do consumo alimentar do Sistema de Vigilância Alimentar e Nutricional e sintomas de depressão e ansiedade baseado no inventário de A Mente Vencendo o Humor. A pesquisa foi aprovada sob parecer $\mathrm{n}^{\circ}$ 4.357.984. Ao todo, 36,5\% dos entrevistados estavam em algum nível de insegurança alimentar e nutricional. A maioria $(93,9 \%)$ apresentou hábitos alimentares satisfatórios durante a pandemia. Relativo à ansiedade, observou-se que 56,2\% dos participantes sentiam-se preocupados na maior parte do tempo e cansados facilmente. Quanto à depressão 50,7\% dos pesquisados sentiam-se cansados, com perda de energia e apresentavam alteração no padrão de sono. Conclui-se que a alimentação dos participantes encontra-se adequada, embora tenha sido verificado que $1 / 3$ das famílias estavam em insegurança alimentar. Verificaram-se sintomas associados à ansiedade e à depressão em um percentual importante da amostra.
\end{abstract}

Palavras-chave: Isolamento social; Padrões alimentares; Segurança alimentar; Vulnerabilidades em saúde.

\begin{abstract}
The pandemic caused by Covid-19 led to isolation and social distance, loss of jobs, changes in family income, changes in eating habits, as well as difficulties in accessing food and the unpredictability that affect the physical and emotional health of individuals and collectivity. In this context, the aim was to evaluate aspects related to food and mental health of the population of Santa Catarina during the Covid-19 pandemic. Data collection was performed by remotely applying a questionnaire from December 2020 to February 2021. The questionnaire was composed of questions that addressed the sociodemographic profile, Brazilian Scale of Food Insecurity, Test - How is your diet? from the Ministry of Health, Food consumption markers form of the Food and Nutritional Surveillance System and symptoms of depression and anxiety based on the inventory of The Mind Overcoming Humor. The research was approved under Opinion No. 4,357,984. In all, 36.5\% of respondents were at some level of food and nutrition
\end{abstract}


insecurity. The majority $(93.9 \%)$ had satisfactory eating habits during the pandemic. Regarding anxiety, it was observed that $56.2 \%$ of the participants felt worried most of the time and easily tired. As for depression, $50.7 \%$ of respondents felt tired, with loss of energy and had an alteration in their sleep pattern. It is concluded that the food of the participants is adequate, although it was found that $1 / 3$ of the families were food insecure. Symptoms associated with anxiety and depression were found in an important percentage of the sample.

Keywords: Social isolation; Food standards; Food safety; Health vulnerabilities.

\section{Resumen}

La pandemia provocada por el Covid-19 generó aislamiento y distanciamiento social, pérdida de puestos de trabajo, cambios en los ingresos familiares, cambios en los hábitos alimentarios, así como dificultades para acceder a los alimentos y la imprevisibilidad que afectan la salud física y emocional de los individuos y la colectividad. En este contexto, el objetivo fue evaluar aspectos relacionados con la alimentación y la salud mental de la población de Santa Catarina durante la pandemia Covid-19. La recolección de datos se realizó mediante la aplicación remota de un cuestionario de diciembre de 2020 a febrero de 2021. El cuestionario fue compuesto por preguntas que abordaban el perfil sociodemográfico, Escala Brasileña de Inseguridad Alimentaria, Test - ¿Cómo es su dieta? del Ministerio de Salud, Formulario de marcadores de consumo de alimentos del Sistema de Vigilancia Alimentaria y Nutricional y síntomas de depresión y ansiedad en base al inventario de La Mente Superando el Humor. La investigación fue aprobada con el dictamen $n^{\circ}$ 4.357.984. En total, el 36,5\% de los encuestados se encontraba en algún nivel de inseguridad alimentaria y nutricional. La mayoría $(93,9 \%)$ tenía hábitos alimentarios satisfactorios durante la pandemia. En cuanto a la ansiedad, se observó que el 56,2\% de los participantes se sentía preocupado la mayor parte del tiempo y se cansaba fácilmente. En cuanto a la depresión, el 50,7\% de los encuestados se sentía cansado, con pérdida de energía y tenía una alteración en su patrón de sueño. Se concluye que la alimentación de los participantes es adecuada, aunque se encontró que 1/3 de las familias padecían inseguridad alimentaria. Los síntomas asociados a la ansiedad y la depresión se encontraron en un porcentaje importante de la muestra.

Palabras clave: Aislamiento social; Normas alimentarias; Seguridad alimentaria; Vulnerabilidades de salud.

\section{Introdução}

Em dezembro de 2019 um surto de uma doença respiratória, causada pelo novo coronavírus (SARS-CoV-2), foi detectado na província de Wuhan, na China. Até março de 2020, o vírus havia se disseminado para centenas de países, causando infecções e óbitos, especialmente em grupos considerados de risco, como indivíduos com comorbidades, idosos e gestantes. A transmissão do vírus ocorre entre humanos principalmente por meio do contato com pessoas infectadas por gotículas respiratórias causando a doença denominada de Covid-19 (Brasil, 2020). Nesse cenário, medidas como a quarentena, isolamento e distanciamento social foram adotadas pelos países para diminuição de transmissibilidade e contenção da pandemia. Na prática de saúde pública, o termo quarentena refere-se à separação de pessoas ou comunidades expostas a uma doença infecciosa. Já o isolamento social, por sua vez, aplica-se à separação de pessoas que estão infectadas e o distanciamento social abrange medidas que buscam restringir o convívio social (Wendy et al., 2020).

Dentre as inúmeras consequências da pandemia, o acesso aos alimentos por parte da população foi dificultado. Assim, tanto as instabilidades sociais quanto as econômicas favoreceram a fome de vulneráveis. O direito humano à alimentação adequada é básico a todos os cidadãos brasileiros e está previsto entre os direitos sociais constitucionais. Neste sentido, a Lei Orgânica de Segurança Alimentar e Nutricional (LOSAN) é um marco no combate à fome, e define a Segurança Alimentar e Nutricional como o direito de todos ao acesso regular e permanente a alimentos de qualidade, em quantidades suficientes, sem comprometer o acesso a outras necessidades essenciais, respeitando a diversidade cultural e que sejam social, econômica e ambientalmente sustentáveis (Brasil, 2006). A Escala Brasileira de Insegurança Alimentar (EBIA) avalia de maneira direta, questões relativas às experiências do indivíduo com relação à fome ao nível domiciliar. Nesta ótica, a EBIA é um instrumento com alta validade para o diagnóstico da (in) segurança alimentar no Brasil e útil também como parâmetro para avaliar a fome durante a pandemia de Covid-19 (IBGE, 2006).

A pandemia também provocou alterações nos aspectos emocionais, sociais e ambientais associados a indivíduos e populações. As mudanças nas escolhas e hábitos alimentares são evidentes nesta situação de incertezas (Asbran, 2020). O isolamento social interferiu negativamente quanto ao acesso aos alimentos in natura, promotores de saúde e por outro lado 
facilitou o consumo de alimentos industrializados por conta da maior validade e praticidade. Ainda, o sedentarismo exacerbado pelo isolamento social e as alterações dos hábitos alimentares, em especial o aumento no consumo de produtos ultraprocessados, sabidamente relacionados à incidência de doenças crônicas não transmissíveis em indivíduos de todas as idades, influenciam negativamente na qualidade de vida da população (Almeida et al., 2018).

Os hábitos alimentares dos indivíduos fazem parte da cultura de cada região (Almeida Júnior et al., 2020). No entanto, a rotina acelerada que envolve as famílias, normalmente é usada como justificativa para a ausência de tempo para hábitos alimentares saudáveis. Neste sentido, preparações práticas que satisfaçam seus desejos de maneira instantânea, sem demandar muito esforço são apreciadas no cotidiano (Brasil, 2014). Porém, pressupõem-se mudanças ainda maiores dessas escolhas alimentares à medida que um contingente importante da população migrou para o isolamento social durante a pandemia de Covid-19. Compreender essas alterações se torna relevante para que as políticas públicas de saúde possam ser planejadas e implementadas para o restabelecimento da população afetada, bem como para atuar de forma preventiva em futuros eventos similares.

Em tempos de pandemia, a saúde física dos indivíduos e o combate ao agente patogênico acabam sendo os focos primários de atenção de gestores, trabalhadores e profissionais da saúde, contudo, as implicações da saúde mental tendem a ser agravadas (Ornell et al., 2020). As emoções são consideradas determinantes das escolhas alimentares (Kaufman, 2012). Indivíduos que sofrem de ansiedade e depressão tendem a ingerir alimentos que satisfaçam momentaneamente suas condições, como é o caso dos alimentos ricos em açúcares. Neste sentido, a depressão, ansiedade e estresse diante da pandemia têm sido identificados na população geral (Wang et al., 2020). Alterações nos aspectos emocionais e nos hábitos alimentares também estão associadas ao quadro socioeconômico da população. Cenários de instabilidade econômica, desemprego e redução da renda familiar provocados pela pandemia podem influenciar diretamente na aquisição de alimentos e nas emoções que por sua vez interferem nas escolhas alimentares de indivíduos e coletividade, levando a um estado de vulnerabilidade (Carrara, 2018).

Neste cenário, estudos referentes às modificações de hábitos alimentares, estado de (in) segurança alimentar e saúde mental da população em decorrência da pandemia de Covid-19 ainda são escassos, contudo, há indícios de repercussões negativas significativas (Schmidt et al., 2020). Desta forma, tornou-se pertinente verificar essas possíveis alterações no estado catarinense, um, dentre aqueles que adotaram políticas mais intensas de isolamento social. Nesse contexto, o estudo buscou a) avaliar o estado de (in) segurança alimentar e nutricional da população catarinense durante a pandemia de Covid-19; b) descrever a frequência e as preferências alimentares da população catarinense durante a pandemia de Covid-19; c) investigar fatores psicológicos que possam estar associados às alterações alimentares dos catarinenses.

\section{Metodologia}

\subsection{Caracterização do estudo}

Trata-se de um estudo de natureza quantitativa de caráter transversal, realizado no período de dezembro de 2020 a fevereiro de 2021, junto à população residente no Estado de Santa Catarina, localizado na região Sul do Brasil.

A população é composta por 7.252.502 habitantes, formada majoritariamente por descendentes europeus. As maiores cidades do estado em população são Joinville, Florianópolis e Blumenau. Segundo o censo de 2010, a densidade demográfica era de 65,27 hab./ $\mathrm{km}^{2}$. O Índice de Desenvolvimento Humano (IDH) dos catarinenses era de $0.774 \mathrm{em} \mathrm{2010,} \mathrm{o} \mathrm{terceiro} \mathrm{maior}$ do país. Em 2018, o Produto Interno Bruto (PIB) atingiu R \$98,2 bilhões, o sexto maior do país e o PIB per capita de R $\$ 42.149$ era o $4^{\circ}$ maior. Em 2020, as exportações atingiram US $\$ 8,1$ bilhões ou 3,9\% do total nacional. Santa Catarina é o terceiro estado importador do Brasil (IBGE, 2020). 


\subsection{Participantes da pesquisa}

Foi utilizado um teste probabilístico para definição do tamanho da amostra com uma margem de erro de $5 \%$ e um nível de confiança de $95 \%$. Foram considerados participantes da pesquisa residentes no estado de Santa Catarina e maiores de 18 anos. Foram excluídos do estudo os sujeitos que não autorizaram por meio do TCLE (Termo de Consentimento livre e esclarecido). O esforço amostral se deu durante de três meses, ou seja, de dezembro de 2020 a fevereiro de 2021.

\subsection{Coleta de dados}

A coleta de dados consistiu na aplicação de um questionário de maneira remota, por meio da plataforma Google forms e divulgado à população catarinense via redes sociais (Facebook, Instagram, WhatsApp e e-mail) dos pesquisadores. O questionário foi composto por questões sociodemográficas, Escala Brasileira de Insegurança Alimentar, Teste - Como está sua alimentação? do Ministério da Saúde, formulário de marcadores do consumo alimentar do Sistema de Vigilância Alimentar e Nutricional, sintomas de depressão e ansiedade baseado no inventário de A Mente Vencendo o Humor.

Quanto ao questionário sociodemográfico, foram abordadas questões como sexo, ocupação e renda familiar. Para a avaliação do nível de (in) segurança alimentar e nutricional foi utilizado o questionário de 14 questões da Escala Brasileira de Insegurança Alimentar (EBIA) como parâmetro para compreender a situação de insegurança alimentar do indivíduo em termos de qualidade e quantidade de alimentos. Esta escala é descrita em quatro categorias, a de segurança alimentar se aplica aos domicílios que possuem acesso regular e permanente aos alimentos em quantidade suficiente e qualidade adequada. Já a insegurança alimentar pode ser dividida em leve, quando há preocupação quanto ao acesso aos alimentos no futuro e a qualidade não é adequada para o consumo. Insegurança moderada quando há redução quantitativa de alimentos entre adultos da família e insegurança grave é a redução quantitativa de alimentos atingindo as crianças da família (Sagi, 2014; Esperandio, Morais \& Priore, 2018).

Como critérios de pontuação e classificação dos resultados, no que tange a segurança alimentar todas as perguntas são respondidas negativamente. $\mathrm{Na}$ insegurança leve, as perguntas de um a cinco são respondidas afirmativamente em domicílios com menores de 18 anos e afirmativas para as perguntas de um a três em domicílios sem menores de 18 anos e neste nível o aspecto mais afetado é a qualidade da alimentação, juntamente com a preocupação de que possa faltar alimento no futuro próximo. Na insegurança moderada, as perguntas de seis a nove são respondidas afirmativamente em domicílios com menores de 18 anos e as perguntas de quatro a cinco são afirmativas em domicílios sem menores de 18 anos, começando a haver restrição quantitativa na alimentação dos adultos da família. E por fim, na segurança grave são respondidas positivamente as respostas das questões de dez a quatorze do questionário em domicílios com menores de 18 anos e afirmativas as respostas das questões de seis a oito em domicílios sem menores de 18 anos, assim, aparece deficiência quantitativa e ao mesmo tempo fome entre crianças da família (Sagi, 2014).

Para a avaliação da ingestão alimentar, utilizou-se dois inquéritos dietéticos retrospectivos. Entende-se por método retrospectivo, a abordagem que coleta informações do passado, tanto imediato quanto remoto. Utiliza estudos epidemiológicos que relacionam dieta e doença, de maneira que se possa classificar grupos populacionais de acordo com o seu consumo alimentar habitual. Identifica indivíduos com padrões extremos de consumo e possibilita o monitoramento de tendências dos comportamentos alimentares ao longo do tempo (Fagioli \& Nasser, 2008). Desta forma, possibilita relacionar a dieta ao estado nutricional do indivíduo e predizer o aparecimento de doenças crônico-degenerativas (Duarte, 2007).

A análise da qualidade da alimentação dos participantes do estudo foi realizada com base no teste - Como está sua alimentação? do Ministério da Saúde. O teste foi feito por meio de 18 perguntas que envolvem a quantidade de frutas, legumes e hortaliças consumidas, frequência da ingestão de leite e derivados, peixes, carnes vermelhas, quantidade de líquido ingerido por dia, entre outras questões. As respostas são baseadas numa soma de pontuação com a seguinte classificação: até 28 pontos 
- Você precisa tornar a sua alimentação e seus hábitos de vida mais saudável; 29 a 42 pontos - Fique atento com sua alimentação e outros hábitos com atividade física e consumo de líquidos e 43 pontos ou mais - Parabéns! Você está no caminho para o modo de vida saudável. Essa avaliação está associada a versão de bolso do Guia Alimentar para a População Brasileira, no formato de "Dez Passos para uma Alimentação Saudável".

Quanto ao consumo alimentar, aplicou-se o formulário de marcadores do consumo alimentar - indivíduos com 5 anos de idade ou mais do Sistema de Vigilância Alimentar e Nutricional - SISVAN. Objetivou-se a identificação da frequência do consumo de alguns alimentos ou bebidas nos últimos sete dias, que estão relacionados tanto a uma alimentação saudável (exemplo: consumo diário de feijão, frutas, verduras) como a práticas pouco recomendadas (exemplo: consumo frequente de alimentos fritos, refrigerantes e guloseimas).

O Inventário de Depressão consiste em um autorrelato de 19 questões destinadas à identificação de sintomas de depressão. O participante avalia cada item de acordo com a intensidade dos sintomas apresentados na última semana. Para a soma dos escores: "Nem um pouco" vale 0 ponto, "Às vezes" vale 1 ponto, "Frequentemente" vale 2 pontos e "A maior parte do tempo" vale 3 pontos. Ao final, somam-se os pontos, resultando no valor do escore. Já o Inventário de Ansiedade consiste em um autorrelato com 24 questões destinadas à identificação de sintomas de ansiedade. O participante classifica cada item de acordo com a intensidade dos sintomas apresentados na última semana. Para a soma dos escores: "Nem um pouco" vale 0 ponto, "Às vezes" vale 1 ponto, "Frequentemente" vale 2 pontos e "A maior parte do tempo" vale 3 pontos. Ao final, soma-se a quantidade de pontos, resultando no valor do escore (Greenberger \& Padesky, 2017).

\subsection{Análise dos dados}

Os dados coletados foram tabulados e organizados em uma planilha utilizando-se o Software Excel for Windows. Para o desfecho realizou-se uma análise estatística exploratória baseada nas frequências, ilustradas em tabelas e gráficos e mantidas as informações que melhor responderam aos objetivos propostos.

A normalidade das variáveis foi verificada por meio do teste Shapiro-Wilk. Para comparar subgrupos das variáveis foram utilizados testes paramétricos ( $\mathrm{e}$ e ANOVA) e não paramétricos (Kruskal-Wallis). A associação entre as variáveis sociodemográficas e preferências, hábitos alimentares e escores de depressão e de ansiedade foram testadas a partir de análises de correlação de Spearmann (rs) e Pearson (r). Foram considerados significativos os testes com valor estatístico de p < 0,05. Os testes foram obtidos com o auxílio do programa estatístico Past (Hammer, Harper \& Rian, 2001). Foi utilizada análise de regressão linear múltipla para testar a relação de causa e efeito entre idade, renda, pontuação EBIA, pontuação do teste da qualidade da alimentação e marcadores do consumo alimentar com a pontuação da ansiedade e da depressão. Para essa análise foi utilizado o software BioEstat v. 5.0.

\subsection{Aspectos éticos}

A pesquisa foi aprovada pelo Comitê de Ética em Pesquisas envolvendo Seres Humanos (CEP) sob parecer $n^{\circ}$. 4.357.984. Os participantes foram esclarecidos quanto aos objetivos da pesquisa, riscos e benefícios, e responderam o questionário por livre adesão, mediante leitura e aceite do Termo de Consentimento Livre e Esclarecido.

\section{Resultados}

Ao todo foram obtidas 197 respostas válidas. A maioria $(82,2 \%)$ dos participantes eram do sexo feminino. A idade média do sexo masculino era de 36,8 anos e do sexo feminino 36,7 anos. A idade mínima foi de 19 anos e a máxima de 70. Em relação a ocupação dos entrevistados, (18,8\%) eram estudantes, seguidos de servidores públicos (10,2\%), professores $(7,1 \%)$ e 
agente de combate às endemias $(7,1 \%)$. A maioria (53,8\%) possuía renda mensal entre 2 e 5 salários mínimos. A EBIA variou significativamente segundo a ocupação e a renda familiar (Tabela 1).

O consumo alimentar da maioria dos participantes $(74,6 \%)$ demanda de algum nível de atenção $(\mathrm{p}=0,006)$. A média da pontuação do consumo alimentar foi maior para os participantes do sexo feminino $(38,6)(\mathrm{p}<0,001)$. Observou-se maior pontuação de consumo alimentar nos participantes com renda familiar maior, contudo não significativo $(\mathrm{p}=0,29)($ Tabela 1$)$.

Tabela 1 - Perfil sociodemográfico e de consumo alimentar de catarinenses durante a pandemia de Covid-19, dezembro de 2020 a fevereiro de 2021.

\begin{tabular}{|c|c|c|c|c|}
\hline Perfil amostra & Contagem (n) & Percentual & Idade (média) & DP \\
\hline \multicolumn{5}{|l|}{ Sexo } \\
\hline Masculino & 35 & 17,8 & 36,8 & 13,4 \\
\hline Feminino & 162 & 82,2 & 36,7 & 12,0 \\
\hline Ocupação & Contagem (n) & Percentual & Média Ebia & Significância \\
\hline Estudante & 37 & 18,8 & 0,33 & \\
\hline Outros & 27 & 13,7 & 1,00 & \\
\hline Servidor público & 20 & 10,2 & 0,40 & \\
\hline $\begin{array}{l}\text { Agente de combate às } \\
\text { endemias }\end{array}$ & 14 & 7,1 & 2,79 & \\
\hline Professor & 14 & 7,1 & 0,36 & \\
\hline $\begin{array}{l}\text { Administrativo/Auxiliar } \\
\text { administrativo }\end{array}$ & 13 & 6,6 & 0,69 & \\
\hline Técnico/Tecnólogo & 9 & 4,6 & 0,11 & \\
\hline Do lar & 8 & 4,1 & 0,25 & \\
\hline Aposentado & 8 & 4,1 & 0,00 & \\
\hline Empreendedor & 7 & 3,6 & 0,86 & $\begin{array}{c}\mathrm{Hc}=33,5 \\
\mathrm{p}=0,02\end{array}$ \\
\hline Agente comunitário de saúde & 6 & 3,0 & 5,17 & \\
\hline $\begin{array}{l}\text { Vendedor/Representante } \\
\text { Comercial }\end{array}$ & 6 & 3,0 & 1,50 & \\
\hline Nutricionista & 5 & 2,5 & 0,33 & \\
\hline Analista & 4 & 2,0 & 0,50 & \\
\hline Administrador & 4 & 2,0 & 0,25 & \\
\hline Enfermeiro & 3 & 1,5 & 1,59 & \\
\hline Secretária & 3 & 1,5 & 1,00 & \\
\hline Gerente & 3 & 1,5 & 0,86 & \\
\hline Motorista & 3 & 1,5 & 0,67 & \\
\hline Médico & 3 & 1,5 & 0,00 & \\
\hline
\end{tabular}




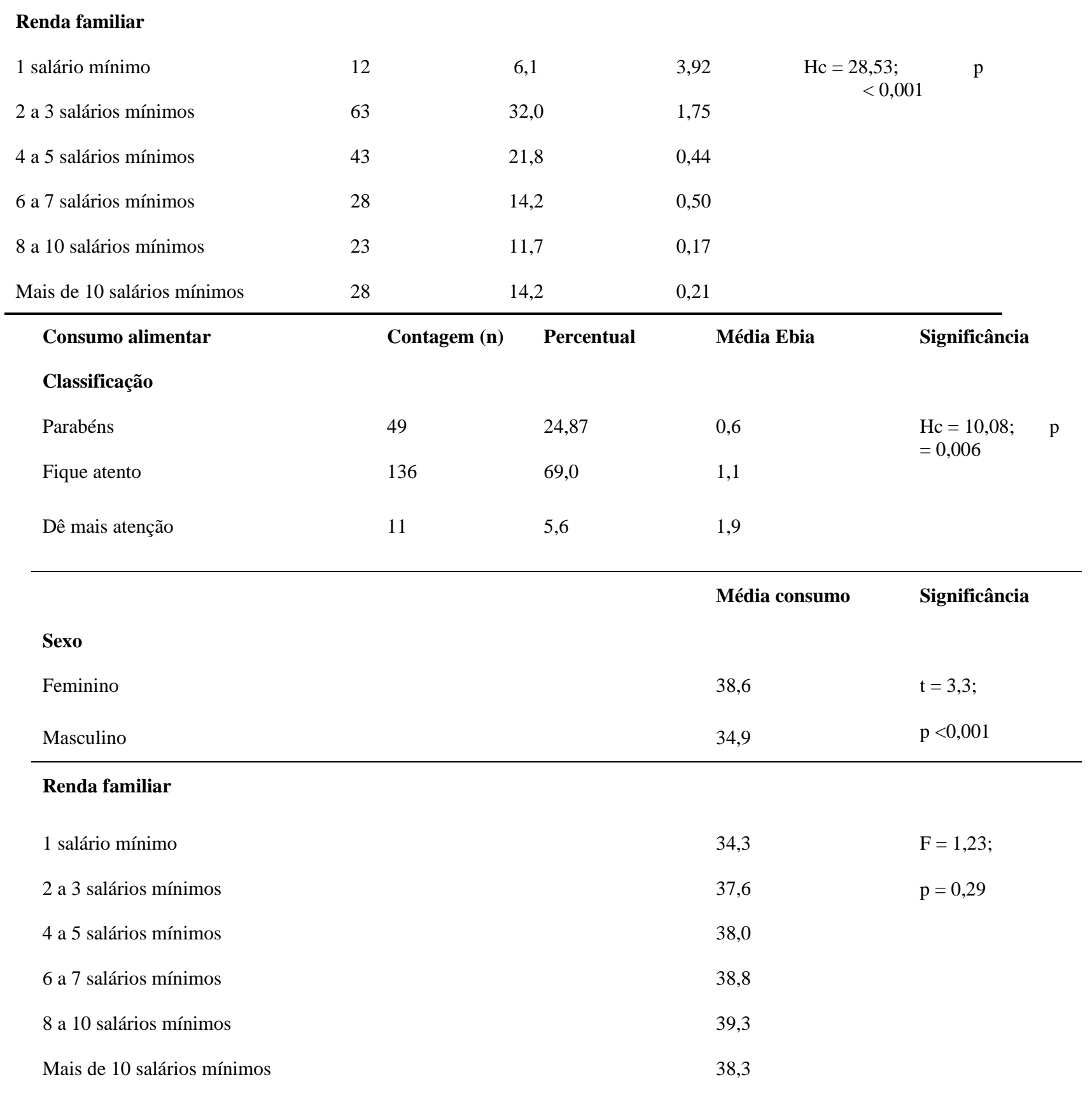

Fonte: Autores (2021).

Observou-se correlação positiva e significativa entre idade e consumo alimentar ( $r s=0,17 ; p=0,02)$. Sobre a correlação entre a pontuação EBIA e idade dos participantes, esta foi negativa e também significativa ( $r s=-0,26 ; p<0,001)$.

Os marcadores do consumo alimentar indicaram que os alimentos de melhor qualidade nutricional (saladas cruas, legumes e verduras, frutas ou saladas de frutas, feijões e lácteos) são consumidos com maior frequência na semana. Já os alimentos considerados de baixo valor nutricional (refrigerantes, biscoitos e doces, biscoitos e salgadinhos e batata e salgadinhos fritos) foram relatados com menor frequência de consumo (Figura 1). 
Figura 1 - Marcadores do consumo alimentar de catarinenses durante a pandemia de Covid-19, dezembro de 2020 a fevereiro de 2021 .

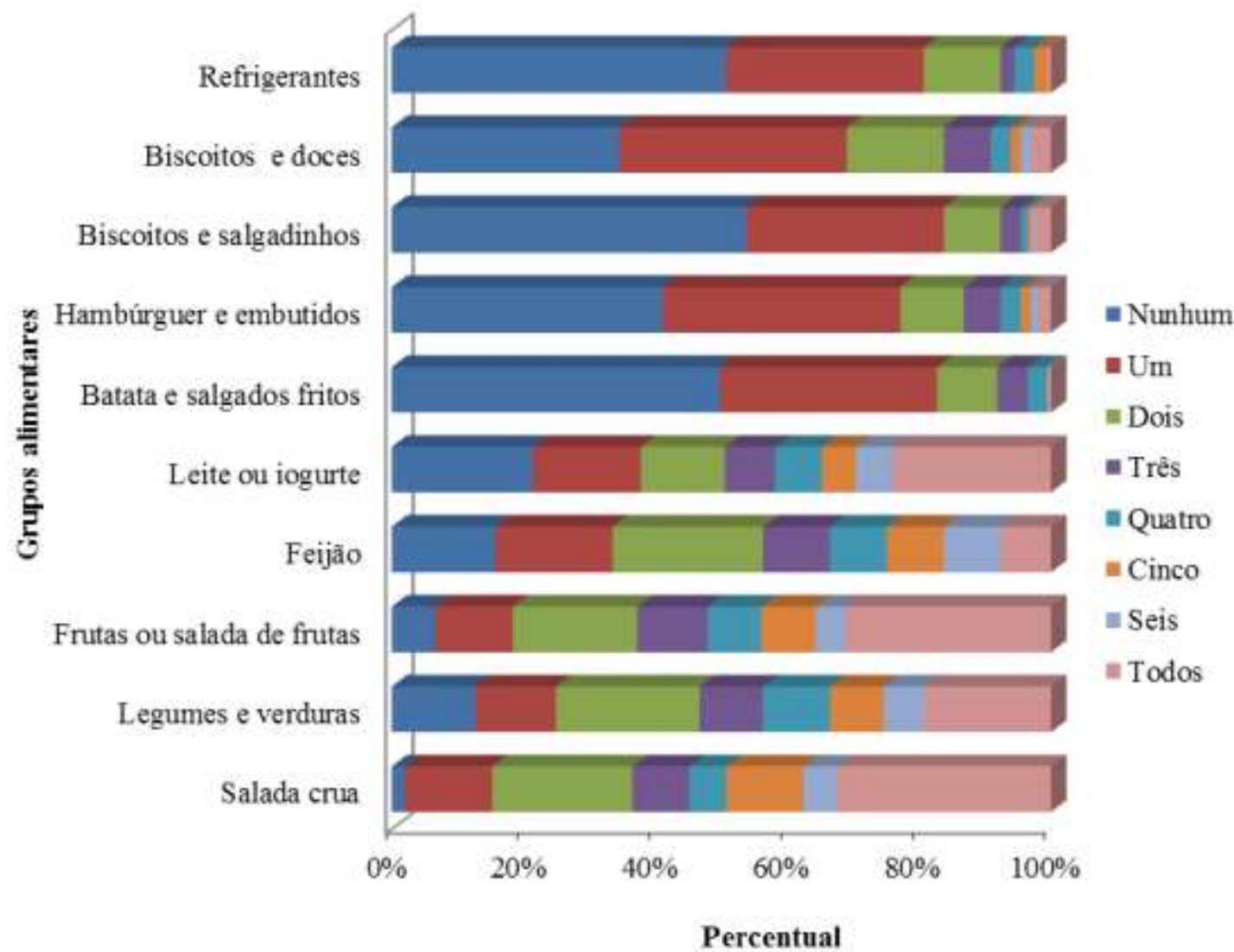

Fonte: Autores (2021).

Ao todo, 63,5\% das famílias estavam em segurança alimentar e nutricional no período de coleta de dados. No entanto, $36,5 \%$ encontravam-se em algum nível de insegurança. Destaca-se que a insegurança alimentar grave esteve presente em 3,0\% dos domicílios (Tabela 2).

Tabela 2 - Classificação de famílias catarinenses segundo a Escala Brasileira de Insegurança Alimentar durante a pandemia de Covid-19, dezembro de 2020 a fevereiro de 2021.

\begin{tabular}{|c|c|c|c|c|c|}
\hline Classificação EBIA & $\mathbf{n}$ & Pontos adultos & $\begin{array}{l}\text { Pontos menores de } \\
18 \text { anos }\end{array}$ & $\begin{array}{l}\text { Soma dos } \\
\text { pontos }\end{array}$ & Percentual \\
\hline Segurança & 125 & 0 & 0 & 0 & 63,5 \\
\hline Insegurança leve & 56 & 73 & 7 & 80 & 28,4 \\
\hline Insegurança moderada & 10 & 41 & 8 & 49 & 5,1 \\
\hline Insegurança grave & 6 & 63 & 28 & 91 & 3,0 \\
\hline Total & 197 & & & & 100 \\
\hline
\end{tabular}

EBIA: Escala Brasileira de Insegurança Alimentar; n: contagem Fonte: Autores (2021). 
A pontuação média da escala de ansiedade foi 24,9 ( $\mathrm{DP}=16,6)$. Observou-se que 31,4\% dos indivíduos sentiam-se preocupados frequentemente ou na maior parte do tempo, 24,8\% sentiam-se cansados facilmente, $21,3 \%$ irritados, $17,8 \%$ relataram tensão e dores musculares, 15,2\% evitam locais onde pudessem ficar ansiosos e 9,6\% sentiam-se incapazes de lidar com as dificuldades (Tabela 3).

A pontuação média da escala de depressão foi 34,4 (DP=14,4). Observou-se que 28,9\%, sentiam-se cansados ou com perda de energia, 21,8\% dos participantes apresentavam alteração no padrão de sono, dificuldade para dormir ou dormindo mais ou menos do que de costume, 19,3\% apresentavam diminuição do desejo sexual, 18,8\% sentiam-se irritados na última semana e 18,3\% apresentavam dificuldade de concentração (Tabela 3).

Tabela 3 - Inventário de ansiedade e depressão “A mente vencendo o humor" de catarinenses durante a pandemia de Covid19, dezembro de 2020 a fevereiro de 2021.

\begin{tabular}{|c|c|c|c|c|}
\hline Ansiedade & $\begin{array}{l}\text { Nem um pouco } \\
\qquad(\mathbf{n} ; \%)\end{array}$ & $\begin{array}{l}\text { Às vezes } \\
(\mathbf{n} ; \%)\end{array}$ & $\begin{array}{l}\text { Frequentemente } \\
(\mathbf{n} ; \%)\end{array}$ & $\begin{array}{l}\text { A maior parte do tempo } \\
\qquad(\mathrm{n} ; \%)\end{array}$ \\
\hline Nervosismo na última semana & $(36 ; 18,3)$ & $(120 ; 0,9)$ & $(32 ; 16,2)$ & $(9 ; 4,6)$ \\
\hline Preocupação na última semana & $(18 ; 9,1)$ & $(117 ; 59,5)$ & $(43 ; 21,8)$ & $(19 ; 9,6)$ \\
\hline Tremores, palpitação, espasmos musculares & $(121 ; 61,5)$ & $(58 ; 29,4)$ & $(17 ; 8,6)$ & $(1 ; 0,5)$ \\
\hline $\begin{array}{l}\text { Tensão muscular, dores musculares, } \\
\text { nevralgia }\end{array}$ & $(80 ; 40,6)$ & $(82 ; 41,6)$ & $(24 ; 12,2)$ & $(11 ; 5,6)$ \\
\hline Inquietação & $(79 ; 40,1)$ & $(84 ; 42,6)$ & $(28 ; 14,2)$ & $(6 ; 3,1)$ \\
\hline Cansaço fácil & $(74 ; 37,6)$ & $(74 ; 37,6)$ & $(38 ; 19,3)$ & $(11 ; 5,5)$ \\
\hline Falta de ar & $(156 ; 79,2)$ & $(28 ; 14,2)$ & $(10 ; 5,1)$ & $(3 ; 1,5)$ \\
\hline Transpiração (não resultante de calor) & $(143 ; 72,6)$ & $(40 ; 20,3)$ & $(10 ; 5,1)$ & $(4 ; 2,0)$ \\
\hline Boca seca & $(138 ; 70,1)$ & $(44 ; 22,3)$ & $(12 ; 6,1)$ & $(3 ; 1,5)$ \\
\hline Tontura ou vertigem & $(149 ; 76,0)$ & $(38 ; 19,4)$ & $(7 ; 3,6)$ & $(2 ; 1,0)$ \\
\hline Náusea, diarreia ou problemas estomacais & $(135 ; 68,5)$ & $(39 ; 20,0)$ & $(19 ; 9,5)$ & $(4 ; 2,0)$ \\
\hline Aumento na urgência urinária & $(155 ; 79,1)$ & $(26 ; 13,3)$ & $(12 ; 6,1)$ & $(3 ; 1,5)$ \\
\hline Rubores (calores) ou calafrios & $(164 ; 83,3)$ & $(27 ; 13,7)$ & $(6 ; 3,0)$ & $(0 ; 0)$ \\
\hline Dificuldade para engolir ou "nó na garganta" & $(155 ; 78,7)$ & $(35 ; 17,8)$ & $(6 ; 3,0)$ & $(1 ; 0,5)$ \\
\hline Sentindo-se tenso ou excitado & $(119 ; 60,4)$ & $(60 ; 30,5)$ & $(15 ; 7,6)$ & $(3 ; 1,5)$ \\
\hline Facilmente assustado & $(140 ; 71,1)$ & $(40 ; 20,3)$ & $(16 ; 8,1)$ & $(1 ; 0,5)$ \\
\hline Dificuldade de concentração & $(81 ; 41,0)$ & $(88 ; 44,7)$ & $(20 ; 10,2)$ & $(8 ; 4,1)$ \\
\hline Dificuldade para adormecer ou dormir & $(98 ; 49,7)$ & $(65 ; 33,0)$ & $(25 ; 12,7)$ & $(9 ; 4,6)$ \\
\hline Irritabilidade & $(59 ; 30,0)$ & $(96 ; 48,7)$ & $(30 ; 15,2)$ & $(12 ; 6,1)$ \\
\hline Evitando lugares onde posso ficar ansioso & $(125 ; 63,5)$ & $(42 ; 21,3)$ & $(19 ; 9,6)$ & $(11 ; 5,6)$ \\
\hline
\end{tabular}


Pensamentos de perigo

Sentindo-me incapaz de lidar com as dificuldades

Pensamentos de que algo horrível irá acontecer
$(151 ; 76,7)$

$(29 ; 14,7)$

$(11 ; 5,6)$

$(6 ; 3,0)$

$(130 ; 66,0)$

$(48 ; 24,4)$

$(9 ; 4,6)$

$(10 ; 5,0)$

$(137 ; 69,5)$

$(45 ; 22,8)$

$(9 ; 4,6)$

$(6 ; 3,1)$

\section{Depressão}

Humor triste ou deprimido na última semana

$(67 ; 34) \quad(107 ; 54,3) \quad(17 ; 8,7) \quad(6 ; 3,0)$

Sentimentos de culpa na última semana

$(110 ; 55,9)$

$(68 ; 34,5)$

$(14 ; 7,1)$

$(5 ; 2,5)$

Humor irritado na última semana

$(43 ; 21,8)$

$(117 ; 59,4)$

$(31 ; 15,8)$

$(6 ; 3,0)$

Menos interesse ou prazer em atividades

costumeiras na última semana

$(77 ; 39,1)$

$(92 ; 46,7)$

$(18 ; 9,1)$

$(10 ; 5,1)$

Afastado ou evitando pessoas

$(99 ; 50,2)$

$(62 ; 31,5)$

$(28 ; 14,2)$

$(8 ; 4,1)$

Achando mais difícil fazer as coisas do que

$(93 ; 47,2)$

$(76 ; 38,6)$

$(21 ; 10,7)$

$(7 ; 3,5)$ de costume

$(149 ; 75,6)$

$(37 ; 18,9)$

$(6 ; 3,0)$

$(5 ; 2,5)$

Dificuldade de concentração

$(82 ; 41,6)$

$(79 ; 40,1)$

$(25 ; 12,7)$

$(11 ; 5,6)$

Dificuldade de tomar decisões

$(83 ; 42,1)$

$(85 ; 43,1)$

$(23 ; 11,7)$

$(6 ; 3,1)$

Pensamentos Suicidas

$(184 ; 93,4)$

$(7 ; 3,6)$

$(4 ; 2,0)$

$(2 ; 1,0)$

Pensamentos recorrentes de morte

$(169 ; 85,8)$

$(19 ; 9,6)$

$(6 ; 3,1)$

$(3 ; 1,5)$

Pensando em um plano suicida

$(187 ; 95,0)$

$(5 ; 2,5)$

$(3 ; 1,5)$

$(2 ; 1,0)$

Baixa autoestima

$(96 ; 48,7)$

$(72 ; 36,5)$

$(21 ; 10,7)$

$(8 ; 4,1)$

Vendo o futuro sem esperança

$(139 ; 70,6)$

$(42 ; 21,3)$

$(9 ; 4,6)$

$(7 ; 3,5)$

Pensamento de autocrítica

$(77 ; 39,1)$

$(88 ; 44,7)$

$(23 ; 11,7)$

$(9 ; 4,5)$

Cansaço ou perda de energia

$(49 ; 24,9)$

$(91 ; 46,2)$

$(41 ; 20,8)$

$(16 ; 8,1)$

Perda de peso significativa ou diminuição do apetite (não inclui perda de peso com um

$(168 ; 85,3)$

$(18 ; 9,1)$

$(7 ; 3,6)$

$(4 ; 2,0)$ plano de dieta)

Alteração no padrão de sono-dificuldade para dormir ou dormindo mais ou menos do que de costume

Fonte: Autores (2021).

A idade apresentou correlação negativa com a depressão. O consumo de alguns alimentos e bebidas se correlacionaram positivamente com a depressão. A renda, a pontuação da frequência alimentar, a frequência do consumo de legumes e verduras, de frutas frescas apresentaram correlação negativa com a ansiedade. Todos os grupos de alimentos avaliados a apresentaram correlação positiva e significativa com o escorre da depressão. A renda, a pontuação da EBIA e a 
frequência de consumo de refrigerantes apresentaram correlação positiva com a pontuação da ansiedade. Ao todo $59 \%$ da pontuação da depressão e $14 \%$ da ansiedade foram explicadas pelos fatores avaliados $(p<0,005)$ (Tabela 4).

Tabela 4 - Relações entre fatores avaliados no estudo (idade, renda, pontuação da Escala Brasileira de Insegurança Alimentar, pontuação da Frequência Alimentar e pontuação do consumo semanal de grupos alimentares (recordatório)) com as pontuações do inventário de ansiedade e depressão (A mente vencendo o humor) de catarinenses durante a pandemia de Covid-19, dezembro de 2020 a fevereiro de 2021.

\begin{tabular}{lcc}
\hline Fatores & \multicolumn{1}{c}{ Depressão } & Ansiedade \\
\hline Idade (anos) & $\mathrm{r}=-0,17 ; \mathrm{p}=0,01^{*}$ & $\mathrm{r}=-0,16 ; \mathrm{p}=0,02^{*}$ \\
Renda (salários mínimos da família) & $\mathrm{r}=0,11 ; \mathrm{p}=0,11$ & $\mathrm{r}=0,21 ; \mathrm{p}=0,003^{*}$ \\
EBIA & $\mathrm{r}=-0,05 ; \mathrm{p}=0,43$ & $\mathrm{r}=0,23 ; \mathrm{p}=0,001^{*}$ \\
Frequência alimentar (QFA) & $\mathrm{r}=0,06 ; \mathrm{p}=0,42$ & $\mathrm{r}=-0,14 ; \mathrm{p}=0,04^{*}$ \\
Salada crua (dias por semana) & $\mathrm{r}=0,49 ; \mathrm{p}<0,001^{*}$ & $\mathrm{r}=-0,12 ; \mathrm{p}=0,09$ \\
Legumes e verdura (dias por semana) & $\mathrm{r}=0,42 ; \mathrm{p}<0,001^{*}$ & $\mathrm{r}=-0,16 ; \mathrm{p}=0,03^{*}$ \\
Frutas frescas (dias por semana) & $\mathrm{r}=0,46 ; \mathrm{p}<0,001^{*}$ & $\mathrm{r}=-0,15 ; \mathrm{p}=0,03^{*}$ \\
Feijão (dias por semana) & $\mathrm{r}=0,37 ; \mathrm{p}<0,001^{*}$ & $\mathrm{r}=-0,11 ; \mathrm{p}=0,12$ \\
Lácteos (dias por semana) & $\mathrm{r}=0,47 ; \mathrm{p}<0,001^{*}$ & $\mathrm{r}=-0,09 ; \mathrm{p}=0,19$ \\
Frituras (dias por semana) & $\mathrm{r}=0,37 ; \mathrm{p}<0,001^{*}$ & $\mathrm{r}=-0,003 ; \mathrm{p}=0,95$ \\
Embutidos (dias por semana) & $\mathrm{r}=0,35 ; \mathrm{p}<0,001^{*}$ & $\mathrm{r}=-0,008 ; \mathrm{p}=0,91$ \\
Biscoitos doces (dias por semana) & $\mathrm{r}=0,33 ; \mathrm{p}<0,001^{*}$ & $\mathrm{r}=-0,02 ; \mathrm{p}=0,78$ \\
Biscoitos salgados (dias por semana) & $\mathrm{r}=0,36 ; \mathrm{p}<0,001^{*}$ & $\mathrm{r}=-0,02 ; \mathrm{p}=0,78$ \\
Refrigerantes (dias por semana) & $\mathrm{r}=0,23 ; \mathrm{p}=0,001^{*}$ & $\mathrm{r}=0,17 ; \mathrm{p}=0,01^{*}$ \\
\hline Total da explicação & $\mathbf{R 2}=\mathbf{0 , 5 9 ;} \mathbf{p}<\mathbf{0 , 0 0 0 1 *}$ & $\mathbf{R 2}=\mathbf{0 , 1 4 ;} \mathbf{p}=\mathbf{0 , 0 0 6 *}$ \\
\hline
\end{tabular}

EBIA: Escala Brasileira de Insegurança Alimentar Fonte: Autores (2021).

\section{Discussão}

A presente pesquisa trouxe como principais resultados: (a) 197 participantes válidos, sendo a sua maioria, do sexo feminino, idade mínima de 19 anos e a máxima de 70, eram estudantes, seguidos de servidores públicos, professores e a maioria com renda mensal entre 2 e 5 salários mínimos; (b) quanto a segurança alimentar e nutricional, a maioria dos participantes $(63,5 \%)$ encontravam-se em segurança e uma parcela significativa $(35,5 \%)$ em algum nível de insegurança alimentar; (c) a maior parte dos pesquisados (93,9\%) apresentaram hábitos alimentares satisfatórios; (d) relativo à ansiedade, observou-se que $(56,2 \%)$ dos participantes sentiam-se preocupados na maior parte do tempo e cansados facilmente e (e) quanto a depressão $(50,7 \%)$ dos pesquisados sentiam-se cansados, com perda de energia e apresentavam alteração no padrão de sono, dificuldade para dormir ou dormindo mais ou menos do que de costume. Esses significados serão ponderados na conclusão.

O perfil sociodemográfico demonstrou que $82,2 \%$ dos participantes da pesquisa eram do sexo feminino, esse predomínio é constatado pelo Censo 2010 que declarou que 50,4\% da população residente em Santa Catarina é do sexo 
feminino. Quanto à ocupação, observou-se que a maioria são estudantes, servidores públicos, professores e profissionais das áreas administrativas. Em Santa Catarina a proporção de catarinenses entre 25 a 34 anos com ensino superior completo em 2017 era de 19,7\%, este dado se relaciona positivamente com o estudo, pois a maioria dos participantes eram estudantes de graduação. A renda prevalente dos participantes da pesquisa foi entre dois a cinco salários mínimos, 53,8\%. Essas características podem ser atribuídas por Santa Catarina ter a menor desigualdade de renda entre os estados brasileiros, pois, a renda média é $25,9 \%$ maior do que a média brasileira e possui a menor proporção da população vivendo abaixo da linha de pobreza, com 8,5\% dos catarinenses vivendo com menos de um salário mínimo (Santa Catarina, 2019).

Ainda relativo ao perfil sociodemográfico da população do estudo, observou-se forte relação desses dados com a rede contato dos pesquisadores, já que o compartilhamento dos questionários foi feito em redes sociais com base em relações pessoais e profissionais dos pesquisadores. A Pesquisa Nacional por Amostra de Domicílios Contínua (PNADC) que demonstrou que em 2019, mais da metade das mulheres $(51,0 \%)$ passaram a ter, ao menos, o ensino médio completo, enquanto entre os homens esse percentual foi de 46,3\%. Ambas as associações vêm aumentando, sendo mais acelerada entre as mulheres e mostrando que há uma melhoria em termos educacionais, renda e participação no mercado de trabalho. Esse cenário tem gerado significativas mudanças na ampliação de autonomia, poder de compra, conhecimento e melhor qualidade de vida (Rodrigues et al., 2021).

O Direito Humano à alimentação adequada é básico a todos os cidadãos brasileiros e está previsto entre os direitos sociais Constitucionais (Brasil, 1988). No entanto, estimativas da Organização das Nações Unidas (ONU), divulgadas em junho de 2020, alertavam que até o final de 2020 mais de 130 milhões de pessoas em todo o mundo poderiam estar em situação de insegurança alimentar, mostrando um agravamento devido à pandemia de Covid-19. Neste cenário, as instabilidades socioeconômicas causadas pelas crises políticas e econômicas dos últimos anos, aliadas a pandemia reforçam as desigualdades e injustiças sociais de grande parte da população brasileira, principalmente aos vulneráveis como moradores de ruas, favelas e áreas periféricas em grandes cidades, no que diz respeito ao acesso regular e permanente aos alimentos em quantidade e qualidade suficientes (Galindo et al., 2021). Contudo, a presente amostra foi composta por profissionais da área da saúde, sendo estudantes, servidores públicos e professores, o que não corrobora com os achados do estudo citado, pois os participantes da pesquisa estão em segurança alimentar na sua maioria, com renda familiar satisfatória e bom nível de escolaridade, garantindo de certa forma maior estabilidade econômica.

O estudo evidenciou que $63,5 \%$ dos entrevistados estavam em segurança alimentar e nutricional no período de coleta de dados. Por outro lado, 36,5\% encontravam-se em algum nível de insegurança e que a insegurança alimentar grave estava presente em 3,0\% dos domicílios. A insegurança alimentar é um processo progressivo, em que geralmente primeiro os adultos começam a se absterem de refeições ou diminuir porções de alimentos e depois de forma mais grave no âmbito familiar, as crianças começam a passar por esta experiência. Neste sentido, um estudo anterior, constatou resultados contrastantes, demonstrando que 59\% dos domicílios inquiridos encontravam-se em situação de insegurança alimentar durante a pandemia e que uma parte significativa dos inquiridos reduziu o consumo de alimentos importantes para a sua alimentação regular, $44 \%$ reduziram a carne e $41 \%$ reduziram o consumo de frutas (Galindo et al., 2021).

O estado de Santa Catarina é economicamente diversificado, organizado em vários polos distribuídos por diferentes regiões. Destaca-se o setor industrial, polo metal mecânico e tecnológico, prestação de serviços, construção civil, turismo, agroindústria entre outros (Santa Catarina, 2019). Não possui grandes metrópoles, pois, a cidade mais populosa, Joinville conta com uma população estimada em 597.658 habitantes, seguido de Florianópolis com 508.826 habitantes (Ibge, 2020). Neste sentido, o estado difere da realidade nacional, pois, como já mencionado, o IDH dos catarinenses era de 0.774 em 2010, renda média maior que a média brasileira e possuem a menor proporção da população vivendo abaixo da linha de pobreza. Desta 
forma, os dados relacionados à renda, explicam em grande parte, porque os catarinenses se encontravam em sua maioria em segurança alimentar e nutricional no período da coleta de dados da pesquisa.

O enfrentamento dos desafios inerentes ao presente cenário requer a articulação e o fortalecimento de instâncias importantes ao diálogo sobre segurança alimentar e nutricional. O planejamento de ações que evitem o desabastecimento, desperdícios e preços não abusivos dos produtos básicos são essenciais para garantir o acesso à alimentação em quantidade e qualidade ideais. A distribuição de alimentos para as populações carentes é uma medida emergencial extremamente necessária para a manutenção da dignidade humana de comunidades em situações de vulnerabilidade durante a pandemia (Alpino et al., 2020). Considerando que houve liberação de auxílio emergencial para a população vulnerável por parte do governo brasileiro no período de coleta de dados, isto é, em 2020 e 2021, este fato pode ter colaborado para a segurança alimentar da população do estado de Santa Catarina naquele momento.

Quanto a correlação entre a pontuação da EBIA e a idade dos participantes, é importante salientar que quanto maior a idade, maior a segurança alimentar dos participantes. A média de idade dos participantes do sexo masculino foi de 36,8 anos e do sexo feminino de 36,7 anos. Santa Catarina destaca-se como maior longevidade do país, com 3,2 anos acima da média nacional, o que tem relação com a qualidade de vida nos municípios catarinenses. Nesta qualidade incluem-se fatores como renda, educação, alimentação, atividade física e acesso a serviços de saúde (Santa Catarina, 2019). Neste sentido, a segurança alimentar e nutricional está relacionada à idade e a realidade do estado que se difere do restante do Brasil.

Quanto aos hábitos alimentares, o estudo mostrou que a maioria (69\%) dos participantes têm hábitos alimentares satisfatórios durante a pandemia de Covid-19, no período da coleta de dados. Porém aponta-se uma atenção além da alimentação, tal como atividade física e consumo de líquidos. A recomendação da OMS é de até cento e cinquenta minutos de atividade moderada por semana, isso inclui atividades no tempo livre, lazer, atividade ocupacional, atividades domésticas, deslocamento para o trabalho ou faculdade (WHO, 2010). Já referente a hidratação, o Guia Alimentar para a População Brasileira reforça que a ingestão de dois litros de água por dia pode ser suficiente para manter a hidratação adequada para a maioria dos indivíduos (Brasil, 2014).

Os participantes da pesquisa informaram que os alimentos de melhor qualidade nutricional (saladas cruas, legumes e verduras, frutas ou saladas de frutas, feijões e lácteos) são consumidos com maior frequência na semana. Já os alimentos considerados de baixo valor nutricional (refrigerantes, biscoitos e doces, biscoitos e salgadinhos e batata e salgadinhos fritos) foram relatados com menor frequência de consumo. Em contrapartida, outros estudos constataram mudanças negativas relacionadas aos hábitos alimentares dos brasileiros durante a pandemia. Nesta lógica, estes estudos reportam que se comparados os hábitos alimentares da população nos anos anteriores, houve durante a pandemia um aumento no consumo de alimentos ultraprocessados e redução na ingestão de frutas e vegetais (Malta et al., 2020; Werneck et al., 2020; Lima et al., 2021).

O perfil da amostra explica os resultados obtidos neste estudo. Perante as adversidades do contexto alimentar e nutricional da população brasileira, bem como as transformações nos hábitos alimentares durante a pandemia, pressupõe-se que por serem participantes com bom nível de escolaridade, profissionais e trabalhadores da área da saúde e serviços públicos, média salarial acima da nacional, satisfatório poder de compra e nível de conhecimento adequado quanto a alimentação e nutrição, estes fatores podem justificar sobremaneira os satisfatórios desfechos deste estudo. Já os índices sociais de Santa Catarina estão entre os melhores do país, pois, o estado tem uma estrutura de saúde descentralizada, desenvolvendo ações no âmbito da gestão do Sistema Único de Saúde (SUS) e por meio de unidades próprias e rede contratualizada. As políticas públicas psicossociais, de alimentação e nutrição são consistentes e apoiadas em todo o território a fim de proporcionar a melhoria das condições de alimentação, nutrição e saúde dos catarinenses (Santa Catarina, 2019). 
Por outro lado, possivelmente tais resultados positivos quanto à alimentação, podem estar relacionados ao aumento do cuidado das pessoas com a própria saúde em tempos de pandemia, já que a alimentação adequada contribui para o fortalecimento do sistema imunológico (Dias et al., 2020). Ademais, pode-se considerar também que nesse período de pandemia as pessoas ficaram mais tempo em casa, em função do distanciamento e ou isolamento social, o que pode ter contribuído para o planejamento alimentar mais adequado, pesquisa por produtos e receitas e/ou compras nutricionalmente mais apropriadas.

Similarmente ao perfil dessa amostragem, um estudo com adolescentes da Espanha, Itália, Brasil, Colômbia e Chile, revelou alterações positivas nos hábitos alimentares. A pesquisa verificou um aumento no consumo de vegetais e redução do consumo semanal de fast-foods (Ruiz-Roso et al., 2020). O Guia Alimentar para a População Brasileira é reconhecido mundialmente e aconselha que se tenha preferência sempre por consumir alimentos in natura ou minimamente processados e preparações culinárias em detrimento aos produtos ultraprocessados (Brasil, 2014). É importante salientar que o consumo de alimentos ultraprocessados está intimamente relacionado ao desenvolvimento de doenças crônicas não transmissíveis (Who, 2010).

Ainda, nesse estudo observou-se correlação positiva e significativa entre idade e consumo alimentar. Isto é, quanto maior a idade, melhores são os hábitos alimentares dos pesquisados. De acordo com os dados da pesquisa de Vigilância de Fatores de Risco e Proteção para Doenças Crônicas por Inquérito Telefônico (VIGITEL), a frequência de consumo regular de frutas e hortaliças é de $34,3 \%$, sendo menor entre homens $(27,9 \%)$ do que entre mulheres $(39,8 \%)$. Em ambos os sexos, essa frequência tende a aumentar com a idade e com o nível de escolaridade (Brasil, 2020).

No decorrer da pandemia, vários fatores impactaram na saúde mental da população, entre eles o medo de contrair a Covid-19, incertezas de como controlar a doença e sua gravidade, transmissão do vírus e até mesmo a morte. Conforme o resultado obtido na escala de ansiedade, a maior parte dos participantes sentem-se preocupados frequentemente ou na maior parte do tempo (31,4\%), isso pode ser explicado pelas incertezas relacionadas à própria saúde e de seus familiares, bem como a renda, já que o isolamento social promoveu rápidas alterações no mercado de trabalho. Houve significativa mudança na rotina das pessoas que afetou as relações familiares, pois, crianças e adolescentes ficaram temporariamente sem frequentar escolas e os adultos tiveram que se adaptar ao trabalho em home office, em consequência disso aumentam as chances de irritabilidade (21,3\%) (Schmidt et al., 2020). Todas essas sensações impactam no padrão de sono, causando dificuldade para dormir ou dormir mais do que de costume.

Uma pesquisa realizada em Curitiba - PR no período de agosto de 2013 a agosto de 2014 demonstrou que em comparação com os homens, as mulheres apresentaram maior prevalência de depressão previamente diagnosticada e praticavam menos atividades físicas, o alto risco de depressão foi mais frequente entre os adultos de menor escolaridade e renda, o que difere do resultado desse estudo, o qual constatou que não houve grande discrepância nas respostas obtidas entre famílias com menor e maior renda mensal, ou seja, a depressão acomete indistintamente a população catarinense (Cavalcanti et al., 2018).

A OMS recomenda que adultos com idade entre 18 e 64 anos devem realizar, pelo menos, cento e cinquenta minutos semanais de exercício físico aeróbio de intensidade moderada, ou pelo menos setenta e cinco minutos semanais de moderada a elevada intensidade, sendo relevante no tratamento e prevenção de depressão e doenças cardíacas, porém para algumas pessoas o isolamento social dificultou essa prática constante, pois houve o fechamento de diversos estabelecimentos por determinação do governo, entre eles as academias e centros de treinamento. Além disso, esportes coletivos também foram temporariamente suspensos e isso afeta diretamente a convivência social. Em contrapartida, algumas pessoas obtiveram mais tempo livre para se dedicar à prática de exercícios individuais, como yoga, ciclismo e alongamento que geram benefícios para a saúde física e 
mental. Propagandas e postagens nas redes sociais apontando a importância da atividade física e orientações para a execução correta, auxiliaram grande parte da população a permanecer ativa (WHO, 2010, Souza \& Graça, 2020).

Estudos realizados em diversos países apontaram aumento do consumo de álcool na pandemia. Essa substância é depressora do sistema nervoso central que, em uma primeira fase, parece relaxar quem o consumiu, mas seu consumo é associado a diversos transtornos mentais. Durante o isolamento, essa associação é potencializada e pode desencadear ou exacerbar episódios depressivos e ansiosos, como também aumentar o risco de suicídio. Outro efeito negativo do consumo do álcool é a violência doméstica, sendo as principais vítimas crianças e mulheres. A violência pode ser qualquer ato que cause morte, dano ou sofrimento físico, sexual, psicológico, patrimonial e moral (Garcia \& Sanchez, 2020; Panagiotidis et al., 2020; Vanderbruggen et al., 2020; Campos et al., 2020).

Os fatores avaliados explicaram $59 \%$ da variação da pontuação da depressão e $14 \%$ da variação da pontuação da ansiedade. Os itens correlacionados positivamente são agentes estressores e os itens com correlação negativa são protetores. Maiores índices de ansiedade e depressão foram identificados nos mais jovens, além disso, quanto maior a EBIA e renda, maior a ansiedade. Portanto, alimentos de baixo valor nutricional, apesar de serem consumidos com menor frequência na semana, é um fator estressor que desencadeia certo nível de ansiedade. A renda e a frequência do consumo de legumes e verduras, de frutas frescas apresentaram correlação negativa com a ansiedade, sendo um agente protetor.

Nesse sentido, dados recentes apontam que a Covid-19 espalhou-se por todos os 56 países e territórios das Américas. Mais precisamente dados de 09 de agosto de 2021, indicam 78,924,997 casos confirmados e 2,034,154 mortes. No mundo, 202,814,435 casos; 4,295,489 mortes; 236 países / áreas / territórios afetados (Paho, 2021). A circunstância atual indica continuidade da pandemia, apesar do esforço de minimizar os impactos com a vacinação. No Brasil a vacinação é vinculada ao Plano Nacional de Operacionalização da Vacinação contra a Covid-19, coordenado pelo Ministério da Saúde, como medida adicional de resposta ao enfrentamento da doença, considerada Emergência de Saúde Pública de Importância Internacional (ESPII) (Brasil, 2021). Os resultados são pontuais, visto que o cenário mudou e está em constante transformação. Recomendase amostras adicionais para avaliar como estes dados estão se comportando.

\section{Conclusão}

Conclui-se que o perfil sociodemográfico do estudo e o período de aplicação dessa pesquisa são fatores determinantes para o desfecho. Desta forma, releva-se o fato de a pesquisa ter se concentrado no município de Chapecó de onde vieram a maior parte das respostas. Reconhecemos que o perfil da amostra é de participantes da área da saúde. Embora as famílias entrevistadas estejam em segurança alimentar durante a pandemia de Covid-19, 1/3 das famílias experienciam em algum nível a insegurança alimentar e nutricional em seus lares. Quanto aos hábitos alimentares, os entrevistados apresentam hábitos satisfatórios quanto ao consumo de alimentos promotores da saúde como frutas, legumes e feijões e insatisfatório quanto ao consumo de água e atividade física. Sobre os fatores psicológicos envolvendo ansiedade e depressão, percebe-se que os entrevistados sofrem de preocupações, irritabilidade, tensões e dores musculares, cansaço fácil, alteração no padrão de sono, diminuição do desejo sexual e dificuldade de concentração durante a pandemia e conforme o resultado do questionário socioeconômico, não houve grande discrepância nas respostas obtidas entre famílias com menor e maior renda mensal, ou seja, a depressão acomete indistintamente a população.

\section{Agradecimentos}

À Universidade Comunitária da Região de Chapecó (Unochapecó). À Bolsa de Pesquisa do Programa PIBIC/CNPq, com recursos do Conselho Nacional de Desenvolvimento Científico e Tecnológico (CNPq), Edital no 17/Reitoria/2020. Ao Programa de bolsas universitárias de Santa Catarina - UNIEDU, ART. 170/CE e Art. 171 - Bolsa de Pesquisa. 


\section{Referências}

Almeida, J., Mendonça, A., Gonçalves, I., \& Batista, L. (2018). Intervenção nutricional através de oficinas culinárias e palestras educativas: aplicação e influência no estado nutricional de adultos. Revista Brasileira de Obesidade, Nutrição e Emagrecimento, 12 , 126-131. http://www.rbone.com.br/index.php/rbone/article/view/668/516

Almeida J., S., Kairala, R., Pereira, A., Costa, G., Cruz, R., Junior, J., Brito, V., Serra, A., Maniglia, F., \& Furtado, R. (2020). COVID-19 e a infecção por SARS-CoV-2 em um panorama geral. Brazilian Journal of health Review, 3(2), 3508-3522. 10.34119/bjhrv3n2-182

Alpino, T. de M. A., Santos, C. R. B., Barros, D. C. de., \& Freitas, C. M. de. (2020). COVID-19 e (in) segurança alimentar e nutricional: ações do Governo Federal brasileiro na pandemia frente aos desmontes orçamentários e institucionais. Caderno de Saúde Pública. 36(8), 1-17. 10.1590/0102-311X00161320

Asbran. (2020). Guia para uma alimentação saudável em tempos de COVID-19. Associação Brasileira de Nutrição. https://www.asbran.org.br/storage/downloads/files/2020/03/guia-alimentar-covid-19.pdf

Bettin, B. (2017). Fatores associados e instrumentos de avaliação da alimentação emocional: uma revisão narrativa da literatura. Trabalho de Conclusão de Curso não-publicado, Departamento de Nutrição, Universidade Federal do Rio Grande do Sul. Porto Alegre, Brasil.

Bezerra, T., Olinda, R., \& Pedraza, D. F. (2017). Insegurança alimentar no Brasil segundo diferentes cenários sociodemográficos. Ciência e saúde coletiva, 22(2), 637-651. 10.1590/1413-81232017222.19952015

Brasil. (2006). Lei 11.346 de 15 de setembro de 2006. Cria o Sistema Nacional de Segurança Alimentar e Nutricional - SISAN com vistas em assegurar o direito humano à alimentação adequada e dá outras providências. Publicado no DOU de 18.9.2006. http://www.planalto.gov.br/ccivil_03/_ato20042006/2006/lei/111346.htm

Brasil. (2014). Guia Alimentar para a população brasileira. Secretaria de Atenção à Saúde. Departamento de Atenção Básica.: Ministério da Saúde.

Brasil. (2020). Protocolo de Manejo Clínico da Covid-19 na Atenção Especializada. Secretaria de Atenção Primária à Saúde. Ministério da Saúde.

Brasil. (2020). Escala Brasileira de Insegurança Alimentar - EBIA: análise psicométrica de uma dimensão da Segurança Alimentar e Nutricional. Secretaria de Avaliação e Gestão da Informação. Brasília, DF: Ministério do Desenvolvimento Social e Combate à Fome.

Campos, B., Tchalekian, B., \& Paiva, V. (2020). Violência contra a mulher: vulnerabilidade programática em tempos de SARS-CoV-2/Covid-19 em São Paulo [Versão eletrônica]. Revista Psicologia \& Sociedade, 32, 1-20. https://www.scielo.br/j/psoc/a/Bqv5dn5fbL3LTrm3PGvJDzN/?lang=pt\&format=pdf

Carrara, C. (2018). Perfis de Aquisição de Alimentos Segundo Grau de Processamento e Fatores Associados em Famílias com Adolescentes de Juiz de Fora $(M G)$. Dissertação de Mestrado, não-publicada, Programa de Pós-Graduação em Saúde Coletiva, Faculdade de Medicina da Universidade Federal de Juiz de Fora. Juiz de Fora, Brasil.

Cavalcanti, A. M., Kusma, S. Z., Chomatas, E. R. V., Ignácio, S. A., Mendes, E. V., Moysés, S. T., \& Filho, R. P. (2018). Noncommunicable diseases and their common risk factors in Curitiba, Brazil: results of a cross-sectional, population-based study. Revista Panam Salud Publica, 42(57), 1-10. 10.26633/RPSP.2018.57

Duarte, A. C. (2007). Avaliação nutricional: aspectos clínicos e laboratoriais. Atheneu, 2007. 607p.

Esperandio, N., Morais, D. de C., \& Priore, S. E. (2018). Escalas de percepção da insegurança alimentar validadas: a experiência dos países da América Latina e Caribe. Ciência e Saúde Coletiva, 23(2), 449-462. 10.1590/1413-81232018232.08562016

Fagioli, D., Nasser, L. A. (2008). Educação nutricional na infância e adolescência: planejamento, avaliação, intervenção e dinâmicas (2a ed.). RCN, 2008.

Galindo, E., Marco A., Melissa D. A., Renata M., Milene P., Larissa M., \& Lúcio R. (2021). Efeitos da pandemia na alimentação e na situação da segurança alimentar no Brasil. Food for Justice Working Paper Series, no. 4. Berlin: Food for Justice: Power, Politics, and Food Inequalities in a Bioeconomy. $10.17169 /$ refubium-29554

Garcia, L. P., \& Sanchez, Z. M. (2020). Consumo de álcool durante a pandemia da COVID-19: uma reflexão necessária para o enfrentamento da situação. Cadernos de Saúde Pública, 36(10), 1-6. 10.1590/0102-311X00124520

Greenberger, D., \& Padesky, C. A. A mente vencendo o humor: mude como você se sente, mudando o modo como você pensa [recurso eletrônico]. (2a ed). Porto Alegre: Artmed, 2017.

Hammer, O., Harper, D. A. T., \& Rian, P. D. (2001). Past: Palaeonthological statistics software package for education and data analysis [Versão eletrônica]. http://palaeo-electronica.org/2001_1/past/issue1_01.htm.

Ibge. (2006). Segurança alimentar no Brasil. 2006. https://agenciadenoticias.ibge.gov.br/agencia-sala-de-imprensa/2013-agencia-de-noticias/releases/13113asi-ibge-traca-perfil-inedito-sobre-seguranca-alimentar-no-brasil

Ibge (2020). IBGE Cidades. https://cidades.ibge.gov.br/brasil/sc/panorama

Kaufman, A. (2012). Alimento e emoção. Revista Abeso, 60, 7-11. https://abeso.org.br/wp-content/uploads/2019/12/60.pdf

Leahy, R. L., Tirch, D. \& Lisa, A. N. (2014) Regulação Emocional em Psicoterapia: um guia para o terapeuta cognitivo-comportamental. Psico-USF, 19(2), 355-357. 10.1590/1413-82712014019002016

Machado, C., Lopes, A., \& Santos, L. (2017). Notificação imprecisa da ingestão energética entre usuários de Serviços de Promoção à Saúde. Ciência \& Saúde Coletiva, 22(2), 417-426. 10.1590/1413-81232017222.21492015 
Research, Society and Development, v. 10, n. 14, e82101421695, 2021

(CC BY 4.0) | ISSN 2525-3409 | DOI: http://dx.doi.org/10.33448/rsd-v10i14.21695

Ornell, F., Schuch, J. B., Sordi, A. O., \& Kessler, F. H. P. (2020). Pandemic fear and COVID-19: mental health burden and strategies. Brazilian Journal of Psychiatry, 42(3), 232-235. 10.1590/1516-4446-2020-0008

Panagiotidis P., Rantis K., Holeva V., Parlapani E., \& Diakogiannis I. (2020). Changes in Alcohol Use Habits in the General Population, during the COVID19 Lockdown in Greece. Alcohol and Alcoholism, 55(6), 702-704. 10.1093/alcalc/agaa092

Pires, J. (2014). Regulação Emocional em Psicoterapia: um guia para o terapeuta cognitivo-comportamental. Universidade Federal de Santa Catarina. Florianópolis, 2014. 166 p.

Rebelo, C. (2018). Relação entre o comportamento alimentar e variáveis motivacionais em crianças e adolescentes. Dissertação de Mestrado, não-publicada, Psicologia Clínica e da Saúde, Universidade da Beira Interior. Covilhã, Brasil.

Rodrigues, L. C. Á., Joia, F. S., Borges, D. G., \& Andrade, J. M. S. (2021). Empreendedorismo feminino: um estudo sobre características, desafios e perfis de gestão de micro e pequenas empreendedoras do Município de Monte Belo-MG. Research, Society and Development, 10. https://rsdjournal.org/index.php/rsd/article/view/13244

Sagi. (2014). Secretaria de Avaliação e Gestão de Informação. Ministério do Desenvolvimento Social e Combate à Fome (MDS). Escala Brasileira de insegurança alimentar - EBIA: Análise psicométrica de uma dimensão da Segurança Alimentar e Nutricional. Estudo técnico n. 01/2014.

Santa Catarina. (2019). Plano Estadual de Saúde 2020/2023. Secretaria de Estado da Saúde de Santa Catarina. Florianópolis, SC.

Schmidt, B., Crepaldi, M. A., Bolze, S. D. A., Neiva, S. L., \& Demenech, L. M. (2020). Saúde mental e intervenções psicológicas diante da pandemia do novo coronavírus (Covid-19). Estudos de Psicologia, 37. 10.1590/1982-0275202037e200063

Souza, M. C., \& Graça, R. L. (2020). Nível de atividade física de praticantes de exercício físico do Sul de Santa Catarina durante a pandemia do Covid-19. Artigo apresentado como trabalho de conclusão de curso, Universidade do Sul de Santa Catarina - UNISUL.

Vanderbruggen, N., Matthys, F., Van, L. S., Zeeuws, D., Santermans, L., Van, D. A. S., \& Crunelle, C. L. (2020). Self-Reported Alcohol, Tobacco, and Cannabis Use during COVID-19 Lockdown Measures: Results from a Web-Based Survey. Eur Addict Res, (26), 309-315. 10.1159/000510822

Wang, C., Pan, R., Wan, X., Tan, Y., Xu, L., Ho, C. S., \& Ho, R. C. (2020). Immediate psychological responses and associated factors during the initial stage of the 2019 coronavirus disease (COVID-19) epidemic among the general population in china. International Journal of Environmental Research and Public Health, 17(5),17-29. 10.3390/ijerph17051729

Wendy, E., Parmet, J. D, Michael, S., \& Sinha, M. D. (2020). Covid-19 - The Law and Limits of Quarantine. The New England Journal of Medicine, 382(28). 10.1056/NEJMp2004211

Zandifar, A., \& Badrfam, R. (2019). Iranian mental health during the COVID-19 epidemic. Asian Journal of Psychiatry, 43, A1-A2. 10.1016 / j.ajp.2019.07.032. 